\title{
Ergodicity of Traffic Flow with Constant Penetration Rate for Traffic Monitoring via Floating Vehicle Technique
}

\author{
Fergyanto E. Gunawan ${ }^{1, \mathrm{a}}$, Bahtiar S. Abbas ${ }^{1}$, Wiedjaja Atmadja ${ }^{1}$, Fajar Yoseph Chandra ${ }^{1}$, Alexander AS Agung ${ }^{1}$, Erwin \\ Kusnandar $^{2}$ \\ ${ }^{1}$ Bina Nusantara University, 11480 Jakarta, Indonesia \\ ${ }^{2}$ Institute of Road Engineering, Department Public Work, 40294 Bandung, Indonesia
}

\begin{abstract}
Traffic congestion in Asian megacities has become extremely worse, and any means to lessen the congestion level is urgently needed. Building an efficient mass transportation system is clearly necessary. However, implementing Intelligent Transportation Systems (ITS) have also been demonstrated effective in various advanced countries. Recently, the floating vehicle technique (FVT), an ITS implementation, has become cost effective to provide real-time traffic information with proliferation of the smartphones. Although many publications have discussed various issues related to the technique, none of them elaborates the discrepancy of a single floating car data (FCD) and the associated fleet data. This work addresses the issue based on an analysis of Sugiyama et al's experimental data. The results indicate that there is an optimum averaging time interval such that the estimated velocity by the FVT reasonably representing the traffic velocity.
\end{abstract}

\section{Introduction}

Traffic congestion in Asian megacities has become extremely worse. Recently, the Indonesia Ministry of Economic Coordination asserted that the inhabitants of Jakarta and greater spend about $60 \%$ of their travel time in the traffic jam. Therefore, reducing the level of congestion is an issue of great interest.

To solve the congestion problem, building an efficient and high capacity mass transportation system is clearly necessary [1]. In addition, implementing Intelligent Transportation System (ITS) has also been demonstrated to be effective [2,3]. In Japan, Electronic Toll Collection (ETC) has completely eliminate congestion ahead of the toll gate and reduce $\mathrm{CO} 2$ emissions by 130000 ton per year [3].

ITS is essentially a combination of the transportation system and the information technology system. The two systems interact in such a way that the transportation can be managed more efficiently. This interaction is possible by means of a set of enabling technologies including data acquisition, data processing, data communication, information distribution, and information utilization [4]. ETC is an excellent example of ITS applications.

Another ITS application is the floating vehicle technique (FVT), which is designed to provide a realtime traffic data or floating car data (FCD) by means of probe vehicles. See Fig. 1 for the FVT framework.

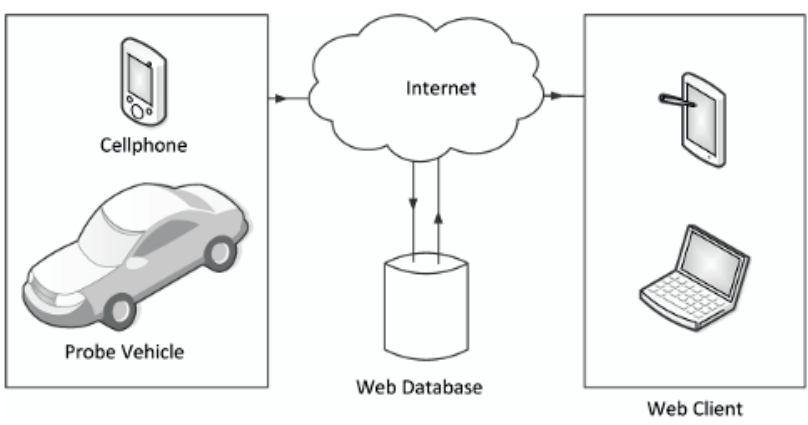

Fig. 1. Framework of traffic monitoring using the floating vehicle technique.

Many publications have been discussing various aspects of FCD [5-14]. Sanwal and Walrand [5] was one of the earliest publications prior the smartphones era. Cathey and Dailey [6] discussed the use of transit vehicles, proposed a Kalman filter to estimate the vehicle position and velocity, compared the estimated velocity to those measured by a speed-trap, and developed a graphical application to display the data in real-time.

Dai et al. [7] studied the use of FVT using a microsimulation model, and evaluated the technique performance in terms of accuracy, reliability, timeliness, and coverage. The accuracy of link speeds was defined as

$$
C_{v}=\operatorname{Prob}\left(\left|\varepsilon_{v}\right| / \varepsilon_{i}\right)
$$

\footnotetext{
$\overline{{ }^{a} \text { Corresponding author: fgunawan@binus.edu, f.e.gunawan@gmail.com }}$
} 
where $\varepsilon_{v}$ is the relative error in link speeds between traffic and probe vehicles, and $\varepsilon_{i}$ a threshold level (5\%, $10 \%, 15 \%$, etc.). Finally, they concluded that the number of probe vehicles was critical for coverage and accuracy, and recommended a penetration rate of $3 \%$ for freeways and $5 \%$ for surface roads, where the penetration rate was defined as the number of probe vehicles during an averaging time interval $\left(t_{a}\right)$ on a traffic link having a flow rate $Q$.

Fabritiis et al. [8] presented a large scale implementation of a traffic management system, so called OCTOTelematics FCD system. At that time, the system received FCD from 600000 private vehicles. The paper also proposed the artificial neural network and pattern matching algorithms for short-term prediction of travel speed. They evaluated algorithm using the traffic on the Roma Ring Road, which has a length of $68.2 \mathrm{~km}$ with $2.4 \%$ penetration rate, 33entries/exists, and 15000 floating cars in workdays.

Uno et al. [9] proposed a method to use transit buses enriched with GPS sensor as probe vehicles. The bus was then used to measure the travel time data, and a procedure was proposed to estimate the associated road travel time.

Liu et al. [10] evaluated the reliability of using taxi dispatch system for real-time traffic information. They found that although among commercial vehicle operations, taxi was the most appropriate for the purpose, but it suffered on a number of issues. Their findings were: Taxi provided good traffic data on high demand links, but the dispatch system could not be a single source of real-time; data from vacant taxis needed careful consideration; GPS location errors had little impact on traffic monitoring, however, the system provided good data for a long road segment.

Herrera et al. [11] was the first field experiment that capable to maintain $2 \%-5 \%$ penetration rate involving 100 vehicles to cover a $10-16 \mathrm{~km}$ long freeway. Campolo et al. [12] developed an integrated smartphone-based platform to acquire, transfer, process, and display traffic and vehicle related data.

Despite of many existing publications and conviction that the accuracy given by the technique depends on the penetration rate, see Eq. 1, and the averaging time interval, none of those publication has quantified and describe how the averaging time interval affects the accuracy of the FVT technique. This article will discuss this critical issue based on well-controlled field experiment where the penetration rate was maintained at a constant rate during the course.

\section{Method}

Essentially, this work relies on the data recorded by Sugiyama et al. [15] during an experiment to produce what is called phantom traffic jam. In their experiment, 22 vehicles were set in a queue along a circular road in a homogeneous lane condition on flat ground. Each driver was requested to maintain a safe distance to its leading vehicle and to cruise, if possible, at $30 \mathrm{~km} / \mathrm{h}$. A 360 degree video camera was set at the center and was used to record the vehicle positions at a rate of one-third of a second. The recorded data are reproduced in Fig. 2.

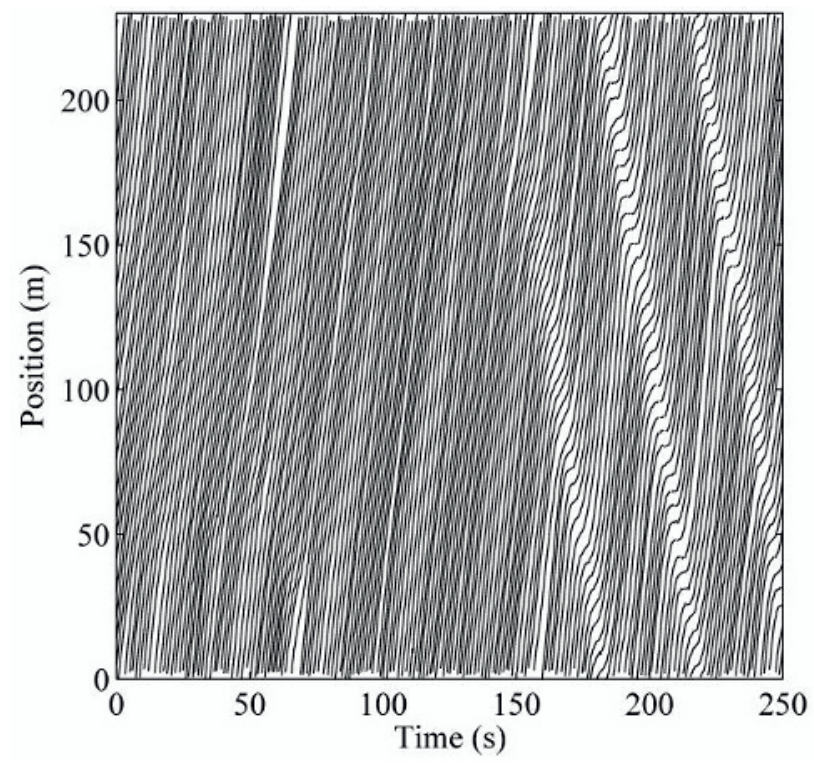

Fig. 2. Space-time plot of movement of a platoon of 22 vehicles in Ref. [15]'s experiment.

Then, the data are unwrapped to obtain a continuous vehicle trajectory in space as shown in Fig. 3. Subsequently, the unwrapped data are smoothed out using the 9-point moving averaging (MA) method to eliminate negative vehicle velocity. Many methods are evaluated including the 5-point and 11-point MA, but the 9-point MA provides the best balance between eliminating the unrealistic negative vehicle velocity and the minimum discrepancy between the smoothed data and the original data.

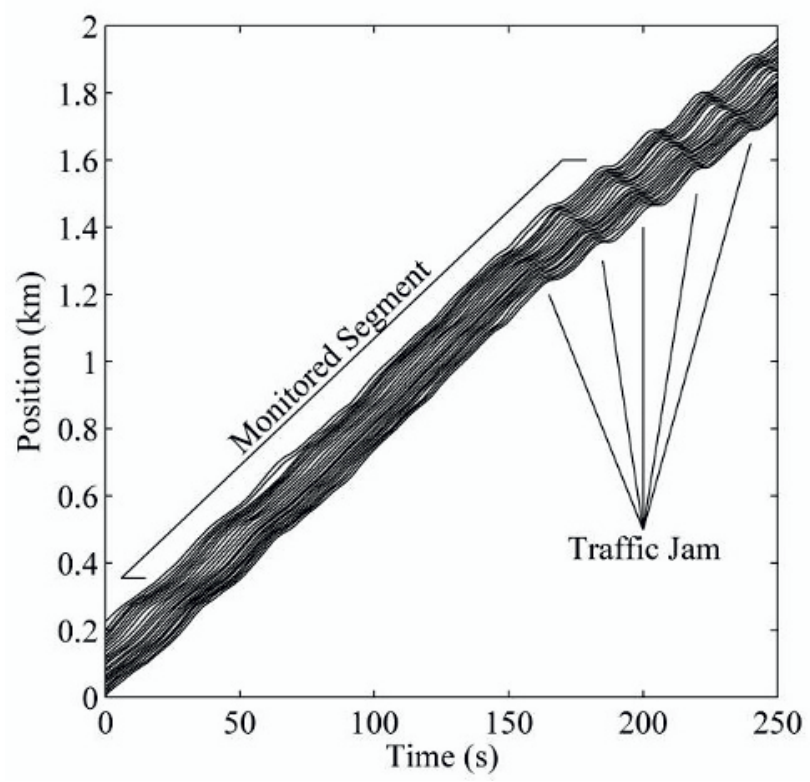

Fig. 3. Unwrapped space-time plot of a platoon of 22 vehicles in Ref. [15]'s experiment. 
Furthermore, the trajectory of each vehicle is fitted using a cubic spline with smoothing [16]; hence, the vehicle velocity can easily be calculated at any instant of time. The smoothing cubic spline is as the following: We consider data $\left(t_{i}, x_{i}\right)$ and $t_{i} \in[a, b]$ for all $i$. We establish cubic spline function $s=s_{p}$ with a smoothing parameter $p$. The function $s$ is differentiable with $m$ derivatives. The smoothing parameter is selected to provide the right balance between the measurement errors:

$$
E(s)=\sum_{i} w_{i}\left|x_{i}-s\left(t_{i}\right)\right|^{2}
$$

where $w_{i}$ is a given weighting, and the smoothness measure:

$$
F\left(D^{m} s\right)=\int_{a}^{b}\left|D^{m} s(t)\right|^{2} d t
$$

The cubic spline function $s(t)$ essentially minimizes:

$$
p \sum_{i} w_{i}\left|x_{i}-f\left(t_{i}\right)\right| 2+(1-p) \int_{a}^{b}\left|D^{m} f(t)\right|^{2} d t .
$$

If we assume installing a loop detector at a point within the monitored segment, see Fig. 3, then the detector can be used to monitor the traffic velocity. The traffic velocity is simply an average of the 22-vehicle instant velocities. These vehicles requires about $33 \sim \mathrm{s}$ duration to cross the loop detector. We denote this velocity $v_{\mu}^{X}$.

In the other hand, in the traffic monitoring using the floating vehicle technique, the traffic velocity is estimated from the velocity history of the probe vehicle. Despite of the difference of the data source, we expect the estimated velocity by the floating vehicle technique $v_{\mu}^{T}$ to reasonably represent the traffic velocity $v_{\mu}^{X}$. We should note that $v_{\mu}^{T}$ depends on the averaging time interval $t_{a}$ that is: a short $t_{a}$ will naturally lead to a highly fluctuating estimated $v_{\mu}^{T}$. On the contrary, a long $t_{a}$ will produce an overly firm estimated velocity.

In this work, we evaluate $v_{\mu}^{T}$ for various $t_{a}$ and describe how the variable affects the estimated velocity.

\section{Ergodicity of a Random Process and Its Relation to Traffic Engineering}

We support the current study with two assumptions. The first is that the average speed of a stream of vehicles on a road is assumed to be a stationary random process. The stationary assumption implies that its statistical properties---for examples, its mean and standard deviation values---will not change across the time.
Mathematically speaking, for a random process $v(x, t)$, then the mean $v_{\mu}^{T}$ can be estimated by

$$
v_{\mu}^{T}=\frac{1}{2 T} \int_{-T}^{+T} v(x, t) d t
$$

The second assumption is: the random process is ergodic, which implies that the above statistical properties can also be obtained by the ensemble average of the process, or:

$$
v_{\mu}^{X}=\frac{1}{2 X} \int_{-X}^{+X} v(x, t) d x
$$

In relation with the traffic engineering, the average speed of a traffic flow that obtained by the loop detector technique is clearly a result of Eq. (6) where the loop detector is implanted across the traffic lanes. Meanwhile, the more modern technique of FVT can easily be used to reduce the speed by Eq. (5).

\section{Results and Analysis}

Firstly, we analyse the platoon dynamic at many positions across the road segment. Those positions are located in a pre-determined range of $[0.36 \mathrm{~km}, 1.60 \mathrm{~km}]$ with a regular spacing of $2.4 \mathrm{~m}$. This region is marked as monitored segment in Fig. 3. The platoon dynamic is represented with its average velocity $\left(v_{\mu}^{X}\right)$ and standard deviation $\left(v_{\sigma}^{X}\right)$ at these positions, and is graphically shown in Fig. 4, In the figure, the upper and lower limits denote $v_{\mu}^{X} \pm v_{\sigma}^{X}$, respectively.

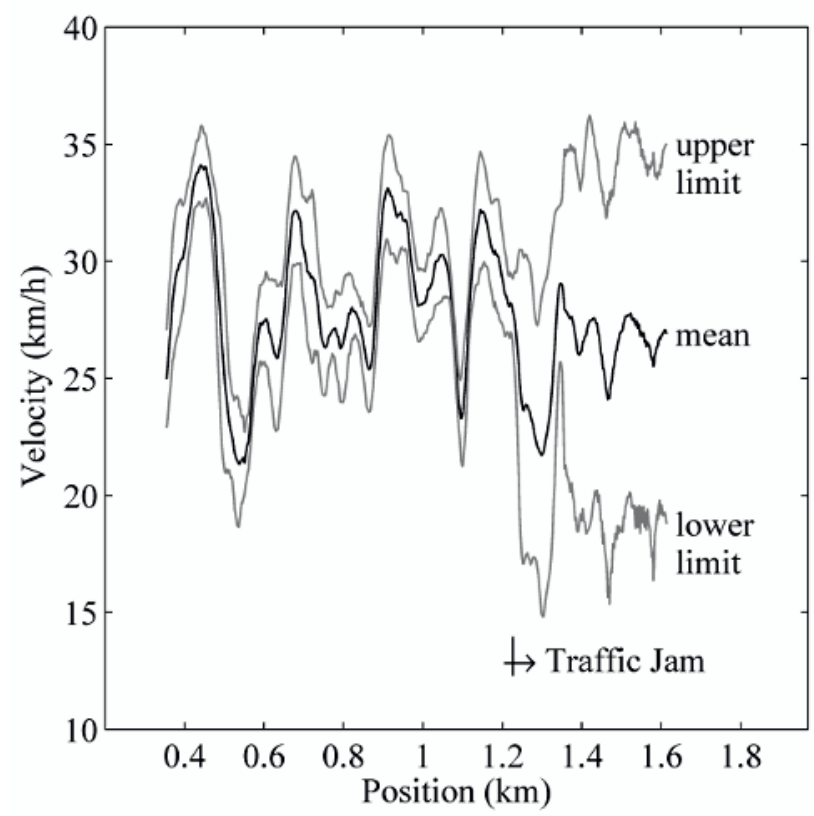

Fig. 4. The platoon average velocity and its variation across the road segment. 
Therefore, the mean velocity in the figure can be regarded as the traffic velocity at the observation point and is usually obtained via loop detector implanted on the road. In this case, the traffic velocity is an average of 22 vehicles, which require about $33 \mathrm{~s}$ to cross a measurement point.

We need to note that in the case in Fig. 4, the phantom traffic jams that occur for a short duration lead to high variation in the individual vehicle velocity. However, prior the jam, the vehicle velocity is relatively uniform across those 22 vehicles. When this fact is related to the traffic measurement using a probe vehicle, we naturally expect that the vehicle will provide a poor prediction when the velocity variation is high and a better prediction when the velocity variation is relatively uniform.

A comparison of the probe vehicle velocity and the platoon average velocity is shown in Fig. 5. The probe vehicle is randomly selected, and the averaging time is varied as $1 \mathrm{~s}, 15 \mathrm{~s}$, and $30 \mathrm{~s}$. However, we limit our analysis prior the onset of the traffic jam.

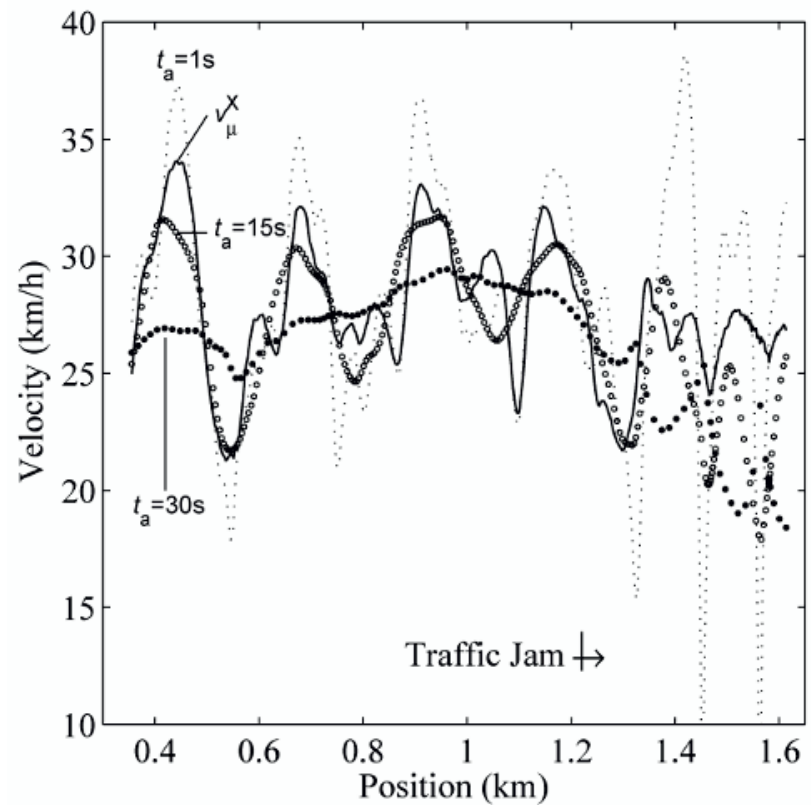

Fig. 5. Comparison of the platoon mean velocity and the probe vehicle velocities at averaging time interval: 1s, $15 \mathrm{~s}$, and $30 \mathrm{~s}$

The figure indicates that prior occurrence of the phantom jam, the discrepancy between the probe vehicle velocity and the platoon average velocity is rather consistent. For $t_{a}=1 \mathrm{~s}$, the probe vehicle overestimates the velocity peaks and underestimate the velocity valleys, consistently. However, for $t_{a}=30 \mathrm{~s}$, the result is on contrary. Figure 5 clearly indicates that there is an optimum averaging time interval $t_{a}^{*}$ that minimizes the relative error (see Eq. 7) of data between the two methods of measurements, and for the present case, we are certain that $1 \mathrm{~s}<t_{\mathrm{a}}^{*}<30 \mathrm{~s}$.

$$
\text { Relative Error }=\frac{\left\|v_{\mu}^{X}-v_{\mu}^{T}\right\|_{2}}{\left\|v_{\mu}^{X}\right\|_{2}} \times 100 \%
$$

Table 1 presents the analysis for various time averaging interval $t_{a}$. A summary is given in Fig. 6 , which shows that the mode of the averaging time interval is $7 \mathrm{~s}$, and the average is about $6.8 \mathrm{~s}$. At this averaging time interval, the estimation error of the FVT is about $4 \%-6 \%$. Figure 7 compares the best estimates of the velocities by the both measurement methods.

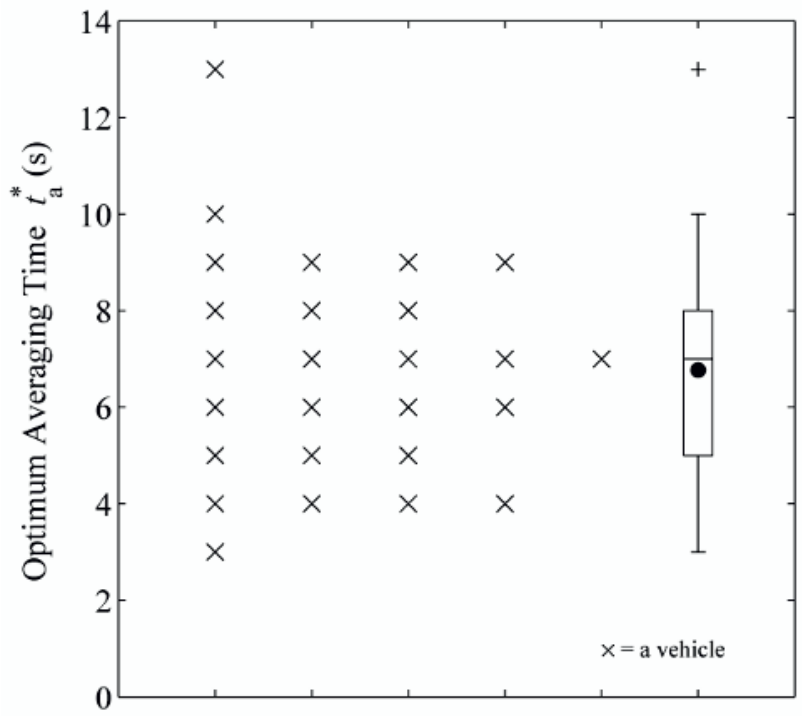

Fig. 6. The optimum averaging time for various probe vehicles; Three vehicles have optimum averaging time interval of $5 \mathrm{~s}$ and $8 \mathrm{~s}$; the median is $7 \mathrm{~s}$, and the mean is $6.8 \mathrm{~s}$

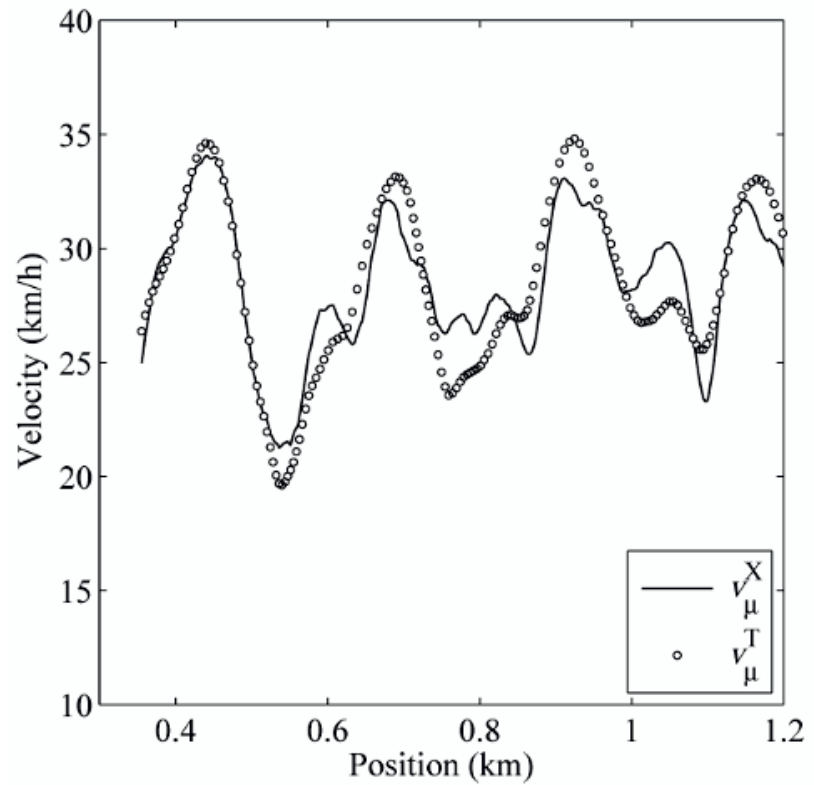

Fig. 7. Comparisons of the platoon mean velocity and the best estimate of the probe vehicle velocity at the averaging time interval $7 \mathrm{~s}$. 
Table 1. The relative error between the probe vehicle velocity and the platoon average velocity for various averaging time interval

\begin{tabular}{|c|c|c|c|c|c|c|c|c|c|c|c|c|c|c|c|c|c|c|c|c|c|c|}
\hline \multirow{2}{*}{$\begin{array}{l}\text { ta } \\
\text { (s) }\end{array}$} & \multicolumn{22}{|c|}{ Vehicle Number } \\
\hline & 1 & 2 & 3 & 4 & 5 & 6 & 7 & 8 & 9 & 10 & 11 & 12 & 13 & 14 & 15 & 16 & 17 & 18 & 19 & 20 & 21 & 22 \\
\hline 1.0 & 6.85 & 9.65 & 12.21 & 9.74 & 8.32 & 8.84 & 6.56 & 5.20 & 5.26 & 5.10 & 5.12 & 4.70 & 5.89 & 5.03 & 5.60 & 7.11 & 6.13 & 6.81 & 5.44 & 5.56 & 5.55 & 7.04 \\
\hline 2.0 & 6.65 & 9.45 & 12.02 & 9.48 & 8.01 & 8.43 & 6.38 & 5.04 & 5.04 & 4.97 & 5.02 & 4.54 & 5.80 & 4.99 & 5.50 & 6.96 & 5.98 & 6.60 & 5.19 & 5.30 & 5.31 & 6.77 \\
\hline 3.0 & 6.38 & 9.19 & 11.74 & 9.10 & 7.58 & 7.85 & 6.14 & 4.85 & .75 & 4.83 & 93 & 4.41 & 70 & 4.96 & .44 & 5.77 & 5.79 & .29 & .84 & 5.01 & 5.04 & .42 \\
\hline 4.0 & 6.11 & 8.90 & 11.39 & 8.65 & 7.06 & 7.17 & 5.88 & 4.67 & 4.45 & 4.72 & 4.87 & 4.34 & 5.61 & 4.96 & 5.39 & 6.56 & 5.59 & 5.93 & 4.49 & 4.71 & 4.77 & 6.05 \\
\hline 5.0 & 5.87 & 8.63 & 11.01 & 8.18 & 6.55 & 6.48 & 5.66 & 4.57 & 4.22 & 4.67 & 4.89 & 4.36 & 5.56 & 4.99 & 5.40 & 6.37 & 5.40 & 5.56 & 4.19 & 4.50 & 4.57 & 5.73 \\
\hline 6.0 & 5.70 & 8.40 & 10.63 & 7.73 & 6.09 & 5.88 & 5.52 & 4.57 & 4.12 & 4.71 & 4.98 & 4.47 & 5.56 & 5.07 & 5.45 & 6.21 & 5.26 & 5.20 & 4.02 & 4.41 & 1.47 & 5.53 \\
\hline 7.0 & 5.61 & 8.23 & 10.27 & 7.35 & 5.74 & 5.42 & 5.49 & 4.71 & 4.18 & 4.85 & 5.16 & 4.65 & 5.62 & 5.20 & 5.56 & 6.11 & 5.19 & 4.92 & 3.99 & 4.43 & 4.47 & 5.44 \\
\hline 8.0 & 5.60 & 8.12 & 9.94 & 7.09 & 5.55 & 5.16 & 5.57 & 4.95 & 4.40 & 5.06 & 5.38 & 4.89 & 5.74 & 5.38 & 5.71 & 6.05 & 5.19 & 4.73 & 4.10 & 4.53 & .56 & 45 \\
\hline 9.0 & 5.63 & 8.07 & 9.67 & 6.95 & 5.53 & 5.11 & 5.74 & 5.28 & 4.74 & 5.35 & 5.65 & 5.16 & 5.91 & 5.60 & 5.89 & 6.06 & 5.26 & 4.68 & 4.31 & 4.70 & 4.72 & 5.53 \\
\hline 10.0 & 5.70 & 8.08 & 9.46 & 6.94 & 5.68 & 5.26 & 5.98 & 5.66 & 5.15 & 5.69 & 5.95 & 5.47 & 6.13 & 5.84 & 6.09 & 6.12 & 5.40 & 4.74 & 4.58 & 4.89 & 4.92 & 5.66 \\
\hline 11.0 & 5.80 & 8.15 & 9.32 & 7.04 & & 5.56 & 6.27 & 6.07 & 0 & 6 & 6.26 & & & 6.11 & 31 & 3 & & 2 & & 1 & 15 & 0 \\
\hline 12.0 & 5.93 & 8.25 & 9.23 & 7.21 & 6.29 & 5.94 & 6.58 & 6.48 & 6.06 & 6.43 & 6.59 & 6.15 & 6.65 & 6.39 & 6.55 & 6.39 & 5.82 & 5.17 & .20 & 5.33 & 5.38 & 5.96 \\
\hline 13.0 & 6.09 & 8.38 & 9.19 & 7.44 & 6.66 & 6.36 & 6.88 & 6.87 & 6.50 & 6.81 & 6.91 & 6.50 & 6.93 & 6.67 & 6.79 & 6.59 & 6.09 & 5.47 & .51 & 5.56 & 5.62 & 6.14 \\
\hline 14.0 & 6.28 & 8.53 & 9.20 & 7.70 & 7.05 & 6.77 & 7.18 & 7.23 & 6.92 & 7.17 & 7.23 & 6.85 & 7.23 & 6.96 & 7.03 & 6.83 & 6.38 & 80 & .81 & 5.80 & .88 & .33 \\
\hline 15.0 & 6.49 & 8.70 & 9.24 & 7.97 & 7.42 & 7.16 & 7.45 & 7.56 & 7.31 & 7.5 & 7.54 & 7.2 & 7.52 & 7.24 & 7.28 & 7.09 & 6.68 & 6.14 & .11 & 6.05 & 5.14 & 6.54 \\
\hline 16.0 & 6.73 & 8.86 & 9.31 & 8.25 & 7.77 & 7.53 & 7.72 & 7.86 & 7.67 & 7.84 & 7.83 & 7. & 7.82 & 7.52 & 7.53 & 7.36 & 6.98 & 6.47 & .40 & 6.31 & 6.41 & 17 \\
\hline 17.0 & 6.97 & 9.03 & 9.41 & 8.54 & 8.11 & 7.87 & 7.97 & 8.14 & 8.00 & 8.15 & 8.12 & 7.86 & 8.10 & 7.79 & 7.78 & 7.64 & 7.29 & 6.80 & 6.69 & 6.59 & 6.69 & 7.01 \\
\hline 18.0 & 7.23 & 9.20 & 9.54 & 8.84 & 8.44 & 8.19 & 8.22 & 8.40 & 8.31 & 8.43 & 8.39 & 8.16 & 8.37 & 8.05 & 8.02 & 7.91 & 7.58 & 7.11 & 98 & 6.88 & 6.97 & 7.25 \\
\hline 19.0 & 7.48 & 9.38 & 9.68 & 9.14 & 8.76 & 8.50 & 8.47 & 8.65 & 8.61 & 8.69 & 8.66 & 8.4 & 8.64 & 8.31 & 8.25 & 8.18 & 7.87 & 7.41 & 7.27 & 7.18 & 7.27 & 7.50 \\
\hline 20.0 & 7.73 & 9.56 & 9.84 & 9.44 & 9.08 & 8.80 & 8.72 & 8.90 & 8.89 & 8.94 & 8.90 & 8.72 & 8.88 & 8.56 & 8.48 & 8.43 & 8.15 & 7.69 & 7.55 & 7.48 & 7.56 & 7.74 \\
\hline 21.0 & 7.98 & 9.74 & 10.01 & 9.74 & 9.39 & 9.08 & 8.97 & 9.14 & 9.16 & 9.18 & 9.15 & 8.97 & 9.12 & 8.80 & 8.71 & 8.68 & 8.41 & 7.96 & 7.83 & 7.79 & 7.85 & 7.98 \\
\hline 22.0 & 8.21 & 9.93 & 10.18 & 1.02 & 9.68 & 9.35 & 9.23 & 9.39 & 9.42 & & 9.38 & & 9.34 & 9.03 & 8.93 & 91 & 8.65 & 1 & 10 & 8.08 & .13 & 8.22 \\
\hline 23.0 & 8.45 & 0.12 & 10.36 & 0.28 & 9.96 & 9.62 & 9.49 & 9.64 & 9.67 & 9.63 & 9.61 & 9.43 & 9.56 & 9.26 & 9.14 & 9.13 & 8.89 & 8.46 & 8.37 & 8.37 & 8.41 & 8.45 \\
\hline 24.0 & 8.68 & 0.31 & 10.54 & 0.52 & 10.22 & 9.87 & 9.75 & 9.89 & 9.91 & 9.85 & 9.83 & 9.65 & 9.77 & 9.49 & 9.36 & 9.34 & 9.12 & 8.71 & 8.64 & 8.66 & 8.68 & 8.68 \\
\hline 25.0 & 8.91 & 10.50 & 10.73 & 0.74 & 10.46 & 10.11 & 10.00 & 10.13 & 10.14 & 10.06 & 10.05 & 9.87 & 9.98 & 9.71 & 9.57 & 9.54 & 9.33 & 8.95 & 8.90 & 8.94 & 8.96 & 8.91 \\
\hline 26.0 & 9.14 & 10.69 & 10.91 & 10.95 & 10.68 & 10.34 & 10.26 & 10.38 & 10.38 & 10.28 & 10.27 & 10.08 & 10.19 & 9.94 & 9.78 & 9.74 & 9.54 & 9.20 & 9.17 & 9.23 & 9.23 & 9.14 \\
\hline 27.0 & 9.38 & 0.89 & 11.09 & 1.14 & 10.90 & 10.57 & 10.52 & 10.61 & 10.60 & 10.49 & 10.49 & 10.30 & 10.39 & 10.16 & 9.99 & 9.93 & 9.74 & 9.45 & 9.44 & 9.51 & 9.51 & 9.38 \\
\hline 28.0 & 9.63 & 11.08 & 11.27 & 11.32 & 11.11 & 10.81 & 10.77 & 10.85 & 10.83 & 10.69 & 10.71 & 10.51 & 10.59 & 10.38 & 10.20 & 10.12 & 9.95 & 9.71 & 9.71 & 9.79 & 9.79 & 9.63 \\
\hline 29.0 & 9.88 & 11.28 & 11.45 & 11.50 & 11.32 & 11.05 & 11.02 & 11.08 & 11.05 & 10.89 & 10.92 & 10.71 & 10.78 & 10.59 & 10.41 & 10.31 & 10.14 & 9.97 & 9.98 & 10.07 & 10.07 & 9.88 \\
\hline 30.0 & 10.15 & 1.47 & 11.63 & 1.67 & 11.53 & 11.29 & 11.27 & 11.30 & 11.27 & 11.08 & 11.12 & 10.92 & 10.98 & 10.80 & 10.61 & 10.50 & 10.34 & 10.24 & 10.25 & 10.34 & 10.34 & 10.15 \\
\hline
\end{tabular}

\section{Conclusions}

The vehicle floating technique has been discussed by many existing publications to be a cost-effective technique to provide a real-time or near real-time traffic information. This article discussed how the averaging time interval affects the accuracy of the FCD. Using data obtained from a well-controlled vehicle stream with a constant penetration rate of $4.6 \%$, we demonstrate that there is an optimum averaging time interval that provides highest accuracy of the traffic velocity. For the current traffic condition, the optimum value is in range of $4 \%-$ $6 \%$. The current recommendation still needs further assessment based on data obtained on various actual traffic conditions.

\section{References}

1. S. Morichi, Journal of the Eastern Asia Society for Transportation Studies 6, 1 (2005)

2. J.D. Nelson, S. Blundell, P. Pettitt, S. Thompson, Transport Review 21, 51 (2001)

3. M. Toshitaka, ed., Japan ITS Handbook 2006-2007 (Highway industry development organization, 2007)

4. K. Chen, J.C. Miles, eds., ITS Handbook 2000: Recommendations from the World Road Association (PIARC) (Artech House, 1999)
5. K.K. Sanwal, J. Walrand, Tech. rep., University of California, Berkeley, California PATH Working Paper UCB-ITS-PWP-95-11 (1995)

6. F. Cathey, D. Dailey, Transit vehicles as traffic probe sensors, in Proceedings IEEE Intelligent Transportation Systems (2001), pp. 579-584

7. X. Dai, M. Ferman, R. Roesser, A Simulation Evaluation of a Real-Time Traffic Information System using Probe Vehicles, in Intelligent Transportation Systems, 2003, Proceedings. 2003 IEEE (2003), Vol. 1

8. C.D. Fabritiis, R. Ragona, G. Valenti, Traffic Estimation and Prediction Based on Real Time Floating Car Data, in $11^{\text {th }}$ International IEEE Conference on Intelligent Transportation Systems (2008)

9. N. Uno, F. Kurauchi, H. Tamura, Y. Iida, Intelligent Transportation Systems 13, 2 (2009)

10. K. Liu, T. Yamamoto, T. Morikawa, Journal of Intelligent Transportation Systems 13, 16 (2009)

11. J.C. Herrera, D.B. Work, R. Herring, X. Ban, Q. Jacobson, A.M. Bayen, Transportation Research Part C: Emerging Technologies 18, 568 (2010)

12. C. Campolo, A. Iera, A. Molinaro, S.Y. Paratore, G. Ruggeri, SMaRTCaR: An integrated smartphonebased platform to support traffic management applications, in First International Workshop on Vehicular Traffic Management for Smart Cities (VTM) (2012)

13. E. Jenelius, H.N. Koutsopoulos, Transportation Research Part B: Methodological 53, 64 (2013)

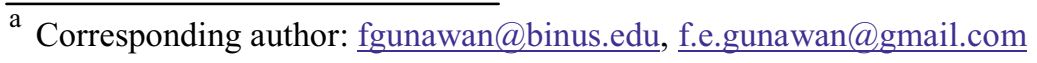


14. M. Rahmani, H.N., Koutsopoulos, Transportation Research Part C: Emerging Technologies 30, 41 (2013)

15. Y. Sugiyama, M. Fukui, M. Kikuchi, K. Hasebe, A. Nakayama, K. Nishinari, S. Ichi Tadaki, S. Yukawa, New Journal of Physics 10, 033001 (2008)

16. C. de Boor, A Practical Guide to Splines (Springer Verlag, 2001)
17. F. De Lillo, F. Cecconi, G. Lacorata, A. Vulpiani, EPL, 84 (2008)

18. L. T. De Luca, Propulsion physics (EDP Sciences, Les Ulis, 2009) 